\title{
The tricky balancing act of using heat shock proteins for cross-presentation
}

\author{
Alice J. Sijts and Willem van Eden* \\ Department of Infectious Diseases and Immunology, Faculty of Veterinary Medicine, Utrecht University, Utrecht, Netherlands \\ *Correspondence: w.vaneden@uu.nl
}

\section{A commentary on}

The role of heat shock proteins in antigen cross presentation

by Murshid, A., Gong, J., and Calderwood, S. K. (2012). Front. Immunol. 3:63. doi: 10.3389/fimmu.2012.00063

Heat shock proteins (HSPs) are evolutionary highly conserved proteins that play a pivotal function in intracellular protein folding and quality control, and thus are essential for maintaining cellular protein homeostasis. It is well-known however that in higher eukaryotes the roles of HSPs are not limited to protein folding. HSPs can activate both the innate and adaptive immune system, with the latter function attributed to their ability to shuttle antigens into both the MHC class I and class II antigen processing pathways. These diverse functions of HSPs have led to their development as vaccine vehicles for tumor immunotherapy. At the same time, the mechanisms underlying the diverse effects of HSPs are poorly understood. Murshid et al. (2012) provide an excellent overview of current knowledge on the pathways contributing to cross-presentation of HSP-associated antigens by MHC class I molecules, and on potential mechanisms supporting the induction of robust CD8 $\mathrm{T}$ cell responses to HSP-delivered antigens. The authors identify different scavenger receptors that are expressed on dendritic cells (DC) and have been shown to bind extracellular HSPs, leading to antigen cross-presentation. They summarize the possible molecular pathways contributing to cross-presentation of scavenger receptor internalized, HSP-delivered antigens by MHC class I molecules, which include the vacuolar or endosome-plasma membrane pathway for short HSP-associated epitopes, as tested for Hsp90, and the endosome to cytosol - cell membrane pathway for larger HSP-associated polypeptides (Ackerman et al., 2003; Cresswell et al., 2005; Rock and Shen, 2005). Interestingly, in accord with the pleiotropic roles of HSPs, especially in the latter antigen processing pathway that is dependent on proteasomes and TAP, the role of HSPs may well extend beyond their use as vehicles to target antigens to the DC, with roles in endosomal escape, and targeting of the antigens to proteasomes being implied. Such a function of HSPs would well fit with numerous other studies, showing a role of endogenous Hsp90 in MHC class I antigen processing, probably by protecting proteasome-released peptides from premature degradation, allowing TAPmediated transport into the ER where they may bind to MHC class I heavy chain/ 及2-microglobulin complexes (Srivastava et al., 1994; Kunisawa and Shastri, 2006). Nevertheless, while it is clear that HSPdelivered antigens can be cross-presented, the triggers leading to immune activation are less well defined. The authors discuss potential mechanisms by which HSPs can create a local inflammatory environment, supporting the induction of robust CD8 T cell responses to HSP-delivered antigens. In the first place, the MHC class I antigen cross-presentation and MHC class II antigen presentation pathway intersect in the endosomal compartments. Thus, HSPdelivered antigens will be processed both for presentation by MHC class I and class II molecules, the last allowing the activation of CD4 T cells which, at least under sterile conditions, are required for DC licensing. In addition, HSPs may function as DAMPs (damage-associated molecular patterns) and engage in interactions with pattern recognition receptors such as TLR2 to stimulate the innate immune system and probably also to further activate the crosspresentation pathway in DC (Amigorena and Savina, 2010). Moreover, HSP-bound scavenger receptor may concentrate in lipid rafts and activate cell signaling pathways, leading for example to NFKB activation.

On the other hand, however, binding of HSPs to another DC-expressed scavenger receptor, SRA/CD204 inhibits immune activation. In addition, HSPs have been shown to be released from cells in exosomes, which may play a role in CD8 T cell cross priming if derived from DC, but have appeared to be immunosuppressive if derived from for example EL4 thymoma cells. Besides the capacity to shuttle antigens into MHC class I and II antigen processing pathways, HSPs have a role as antigens seen by the adaptive immune response. The latter feature of HSPs seems to include the induction of self-HSP cross-reactive $\mathrm{T}$ and $\mathrm{B}$ cell responses triggered by the relatively immuno-dominant microbial HSPs such as those of the HSP60 and HSP70 chaperone families. The inflammation dampening effects of microbial HSPs that were seen in models of experimental autoimmunity were found to relate to induction of regulatory $\mathrm{T}$ cells capable of targeting the upregulated self-HSPs in inflamed tissues. The outcome of clinical trials testing HSP peptides in patients with diabetes and arthritis has lent further support to the immune dampening effects of HSPs (van Eden et al., 2005, 2012; Aldridge, 2012).

Taken together, while it is clear that HSPs deliver antigens to DC for presentation by both the MHC class I and class II molecules, the effects on the activation status of the immune system may as well be negative as positive, with the exact outcome probably depending on a delicate balance of signals received through different receptors on the DC and immune imprints of previous adaptive immune responses. This said, it also becomes easier to appreciate the fact that HSPs are used in cancer therapy, to activate tumor-specific CD8 T cell responses, but also are being developed for immunotherapy strategies aimed to dampen immune responses, for treatment of autoimmune disease. As concluded by the authors, more research especially into the signaling events associated with the immunogenic but also non-immunogenic effects of HSPs will be needed. This to develop these further as highly effective and multi-functional vaccine vehicles. 


\section{REFERENCES}

Ackerman, A. L., Kyritsis, C., Tampe, R., and Cresswell, P. (2003). Early phagosomes in dendritic cells form a cellular compartment sufficient for crosspresentation of exogenous antigens. Proc. Natl. Acad. Sci. U.S.A. 100, 12889-12894.

Aldridge, A. (2012). Toll-like receptor blocker slows beta cell death in type 1 diabetes. Nat. Biotechnol. 30, 124.

Amigorena, S., and Savina, A. (2010). Intracellular mechanisms of antigen cross-presentation in dendritic cells. Curr. Opin. Immunol. 22, 109-117.

Cresswell, P., Ackerman, A. L., Giodini, A., Peaper, D. R., and Wearsch, P.A. (2005). Mechanisms of MHC class I-restricted antigen processing and cross-presentation. Immunol. Rev. 207, 145-157.

Kunisawa, J., and Shastri, N. (2006). Hsp90 alpha chaperones large C-terminally extended proteolytic interme- diates in the MHC class I antigen processing pathway. Immunity 24, 523-534.

Murshid, A., Gong, J., and Calderwood, S. K. (2012). The role of heat shock proteins in antigen cross presentation. Front. Immunol. 3:63. doi: 10.3389/ fimmu.2012.00063

Rock, K. L., and Shen, L. (2005). Cross-presentation: underlying mechanisms and role in immune surveillance. Immunol. Rev. 207, 166-183.

Srivastava, P. K., Udono, H., Blachere, N. E., and Li, Z. (1994). Heat shock proteins transfer peptides during antigen processing and CTL priming. Immunogenetics 39, 93-98.

van Eden, W., Spiering, R., Broere, F., and van der Zee, R. (2012). A case of mistaken identity: HSPs are no DAMPs but DAMPERs. Cell Stress Chaperones 17, 281-292. van Eden, W., van der Zee, R., and Prakken, B. (2005). Heat shock proteins induce $\mathrm{T}$ cell regulation of chronic inflammation. Nat. Rev. Immunol. 5, 318-330.

Received: 16April 2012; accepted: 21 April 2012; published online: 10 May 2012.

Citation: Sijts AJ and van Eden W (2012) The trickybalancing act of using heat shock proteins for cross-presentation. Front. Immun. 3:114. doi: 10.3389/fimmu.2012.00114 This article was submitted to Frontiers in Antigen Presenting Cell Biology, a specialty of Frontiers in Immunology. Copyright (c) 2012 Sijts and van Eden. This is an openaccess article distributed under the terms of the Creative Commons Attribution Non Commercial License, which permits non-commercial use, distribution, and reproduction in other forums, provided the original authors and source are credited. 